\title{
CHARACTERISTICS OF THE NORMAL ELECTROENCEPHALOGRAM. I. A STUDY OF THE OCCIPITAL CORTICAL POTENTIALS IN 500 NORMAL ADULTS ${ }^{1}$
}

\author{
BY MARY A. B. BRAZIER AND JACOB E. FINESINGER \\ (From the Department of Neuropsychiatry, Harvard Medical School, and the Psychiatric Department \\ and Electroencephalographic Laboratory, Massachusetts General Hospital, Boston)
}

(Received for publication August 2, 1943)

This study represents an attempt to codify the main characteristics of the electroencephalogram in the normal adult. This attempt was inspired by the recent interest in the use of the electroencephalogram as a possible method of grading normals in the selection of air pilots.

The chief characteristics of the normal electroencephalogram may be studied by considering the following components:

(1) Dominant frequency .... which is here defined as the frequency in cycles per second of the majority of the waves present. The degree of fluctuation in the dominant frequency of an individual in repeated recordings has also been studied.

(2) Percentage time alpha . . . the percentage of the record occupied by waves of 8.0 to 13.0 cycles per second, whether occurring singly or in chains.

(3) Percentage time intermediate frequencies ... the percentage of the record occupied by waves in the intermediate band (13.5 to 17.5 per second).

(4) Percentage time beta . . . the percentage of the record occupied by cortical potentials of frequencies above 17.5 per second, and of voltages so low as to make them individually uncountable.

(5) Percentage time slow activity . . . i.e., waves slower than 8.0 cycles per second.

(6) Voltage ... In this study, the voltage characteristic studied was the maximum voltage of the potentials from the bipolar occipital leads.

The characteristics listed above will be found to vary in the same person, according to the part of the head examined. Throughout the present study, all analyses were made from bipolar recordings from the occiput.

\footnotetext{
1 This study was aided by a grant from the Harrington Fund.
}

\section{METHOD OF ANALYZING THE RECORDS}

After many attempts at easier and more rapid methods of analysis, the method finally chosen for this research, because it gave more information than any other, was the frequency distribution of the waves, compiled by counting the percentage time covered by waves of each different number of cycles per second.

In order to compile a frequency distribution curve, a 2-minute record, taken when the subject was lying quietly and breathing normally, is first inspected for the presence of artifacts. Any portion showing artifacts due to eyeblinks, muscle movements, etc., is omitted from the sample for analysis. The remainder is measured for total length of time, and this figure becomes the total on which all percentages are calculated.

A transparent grating (designed by Davis), marked off in intervals equivalent to each of the frequencies, is then laid on the record, and the frequency of any chains of waves is thus easily determined. The time covered by waves of each frequency is then totalled, the results being expressed as percentages of the whole period measured.

This process can be shortened by measuring only chains in which at least 3 waves of the same frequency occur together; in the majority of normal records, this arbitrary rule gives an adequately representative picture of the record, although its only specific merit is as a time saver.

When these figures have been compiled, they can be presented either in tabular form, or in diagrammatic form (Figure 1). On the whole, the diagram is to be preferred, because it gives an immediate representation of the most characteristic features of the electroencephalogram.

The characteristics listed in the introduction were studied in detail in a series of $\mathbf{5 0 0}$ young normal adults and the records of these subjects form the basis for the major portion of this paper. Most of these subjects received only one test, but in order to study the consistency of various of the properties of the electroencephalogram, 45 of the series were given 4 to 5 tests each; in all, 176 tests were made on this smaller series of 45 .

\section{RESULTS}

\section{(1) Dominant frequency}

(a) Types of dominant frequency. On first inspection, the most outstanding feature of an electroencephalogram is the dominant rhythm, 


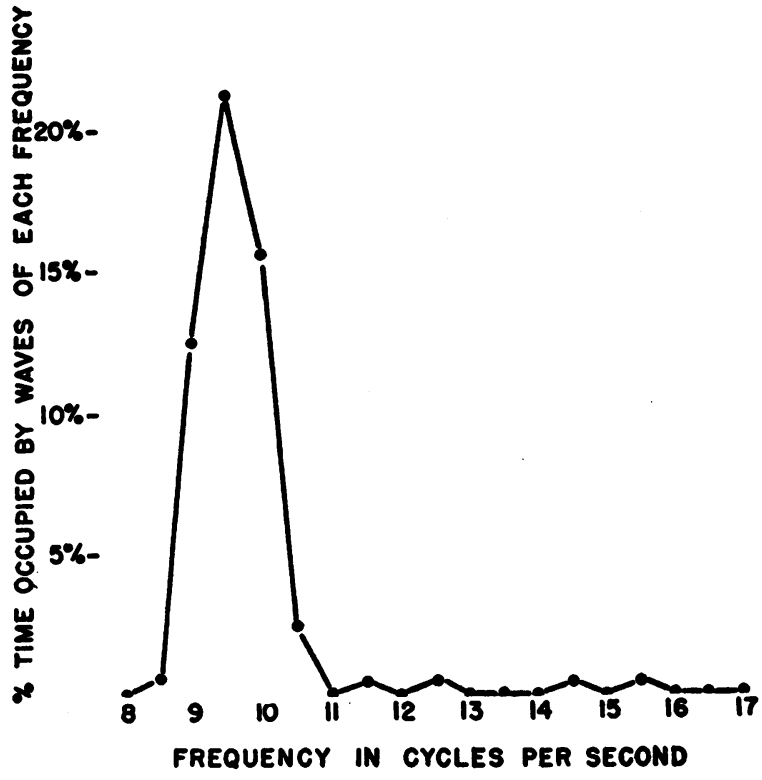

Fig. 1. Graph Compiled from"The"Electroencephalogram of ONe Normal AdUlTSHowing"THE"Percentages of Each Frequency Present, Omiting the_Beta RANGE

The dominant frequency is defined as the one present in the greatest amount, i.e., at the mode of the curve. (In this case, it is 9.5 cycles per second.)

i.e., the frequency in cycles per second which is present in greater amounts than any other frequency. This frequency is usually apparent on rough inspection and most electroencephalographers have adopted the classification into two groups, as first suggested by Berger, namely, the alpha and the beta types. These have usually been defined as the frequencies between 8.0 and 13.0 cycles per second for the alpha group, and frequencies faster than 18.0 cycles for the beta group. This classification is not satisfactory since, in fact, all electroencephalograms consist of a mixture of these rhythms in some degree, and no record consists wholly of either alpha or beta waves. Hence, if this classification is to be used, some criterion must be defined as to the percentage of beta activity which must be present in a record before that record should be classified as a beta type. For the purposes of the present study, records are classed as beta type only if there is present less than 20 per cent of other activity, i.e., of waves slower than 18 cycles per second.
Also, in the present work, a third classification is used, since it has been found in this series of 500 normal adults that there is evidence for regarding the dominant frequencies in the intermediate band (13.5 to 17.5 cycles per second) between the alpha and the beta ranges as a separate entity (Figure 2).

A detailed statistical analysis was made of the activity slower than beta (i.e., slower than $\mathbf{1 8 . 0}$ cycles) in the records of 500 normal adults. The mean for all the dominant frequencies in this range was 10.5 cycles per second, with a standard deviation of 0.9. Thus, any dominant frequency slower than 8.0 or faster than $\mathbf{1 3 . 0}$ cycles is outside 3 times standard deviation for normals, and is therefore, by definition, excluded from the alpha range. Further reason for regarding records with a dominant frequency in the intermediate range as a separate group is found in a study of the distribution curve of the dominant frequencies of 500 normal adults (Figure 3).

In this graph, there appears to be a normal distribution curve dominating the picture but with some outlying stragglers in the faster frequencies. The main curve consists of 474

ALPHA RHYTHM

8.0 TO 13.0 PER SECOND

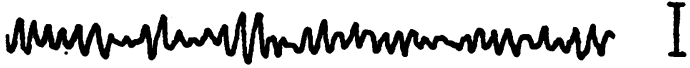

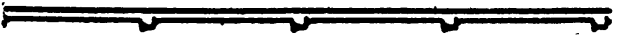

INTERMEOLATE RHYTHM I3.5 TO 17.5 PER SECOND

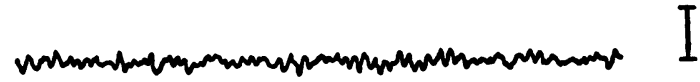

EETA RHYTHM

18.0 TO 35.0 PER SECOND

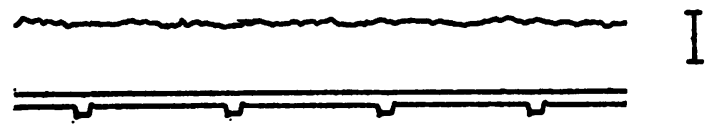

Fig. 2. Types of Normal Electroencephalograms

The upright line to the right of each tracing is the calibration for $100 \mathrm{mV}$. 
subjects whose dominant frequencies give a normal distribution around a mode of 10.0 cycles per second, with 26 subjects outside the normal distribution curve. Hence, it is felt that those frequencies in the range 13.5 to 17.5 should not be included in the alpha group. Frequencies falling within this range are referred to as intermediate rhythms.

In this group of 500 normal adults, examined in this laboratory, the following distribution of the three types (alpha, intermediate, and beta) was found:

$\begin{array}{lrc}\text { Number of subjects: } & 500 & \text { per cent } \\ \text { Number of alpha type (8 to } 13.0 \text { cycles } & 474 & 94.8 \\ \text { per second): } & & \\ \text { Number of intermediate (13.5 to 17.5): } & 18 & 3.6 \\ \text { Number of beta type (18.0 and over): } & 8 & 1.6\end{array}$

(b) Consistency of the dominant frequency. That the dominant frequency remains constant within narrow limits for the same individual over long periods of time can be demonstrated by repeated observations on the same person. A discussion of some factors which may, in certain circumstances, alter the dominant frequency will be reported in a subsequent paper.

Electroencephalograms repeated on the same subjects over a period of a few years revealed only small fluctuations in the dominant frequency from one test to the next:

\begin{tabular}{lccr} 
Subject 1, aged 35, female & \multicolumn{2}{c}{ Subject 2, aged 24, female } \\
July 1940 & 9.5 & Oct. 1940 & 9.5 \\
Dec. 16, 1940 & 9.5 & June 1941 & 10.0 \\
Dec. 26, 1940 & 9.5 & April 1942 & 10.0 \\
Sept. 1942 & 9.5 & Sept. 1942 & 10.0 \\
& Subject 3, aged 36, female & \\
& Dec. 1940 & 20.0 & \\
& June 1941 & 20.0 & \\
& July 1941 & 18.0 & \\
& Aug. 1941 & 20.0
\end{tabular}

This degree of fluctuation is of the same order as that reported by other workers (Loomis, Harvey, and Hobart (1), Jasper and Cruikshank (2), and Jasper and Andrews (3)).

That there is also only a small fluctuation in the dominant frequency of an individual when examined several times during the same day, has been established on a larger group.

One hundred and seventy-six observations were made on 45 normal subjects, all of whom were examined at non-fasting blood sugar levels (above $70 \mathrm{mgm}$ per $100 \mathrm{cc}$.), i.e., 4 to 5 tests were made on the same individual at intervals during the same day.

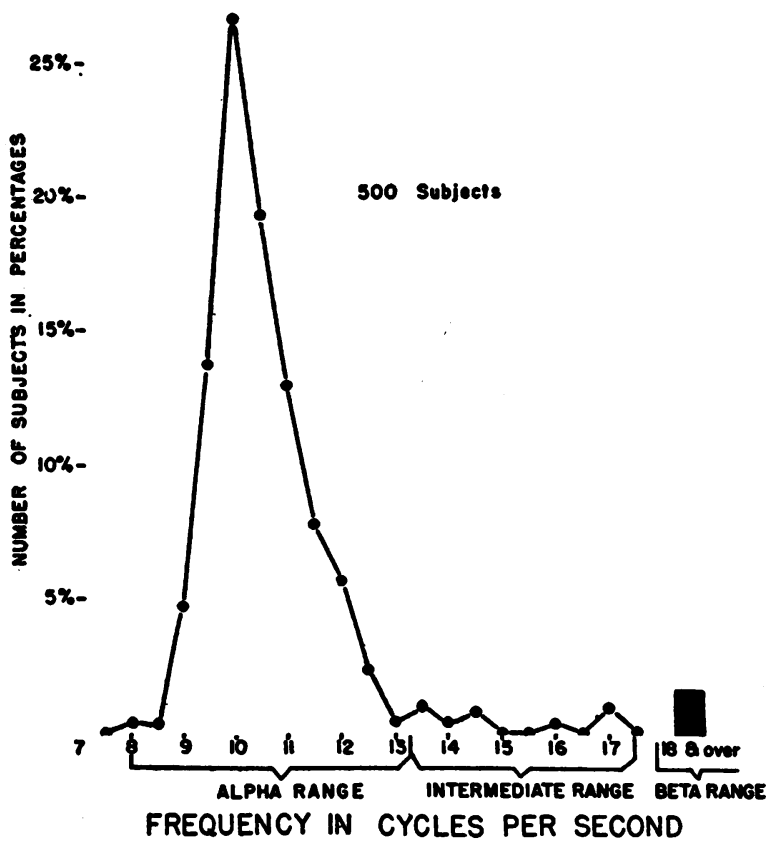

Fig. 3. The Distribution Curve of the Dominant Frequencies Found in the Occipital Rhythis of 500 Normal Adults

Ten of the 45 subjects showed no variation in their dominant frequency. In 1 subject only did the dominant frequency vary from his own mean value by more than 7 per cent. The mean variation for the whole series of 45 subjects was under 1 per cent (176 observations).

(c) Relation between consistency of the dominant frequency and age. If the consistency of a person's dominant frequency be studied, it is found that age is a factor in the degree of variability found in the electroencephalogram at non-fasting blood sugar levels (above $70 \mathrm{mgm}$ ).

This degree of variability is determined by finding the coefficient of variation for the dominant frequency of each individual. (The coefficient of variation $=100 \times \frac{\text { standard deviation }}{\text { mean }}$.)

A group of 45 young adults between the ages of 17 and 38 were thus examined for consistency of. dominant frequency at normal blood sugar levels. By rough observation, it appeared as though the dominant frequency in records of those subjects over the age of 20 was less stable than of those under that age, and for this reason the division into two groups was made at this age level. In the series examined, there were 
13 subjects under the age of 20, and 32 were aged 20 or over. Calculations of the coefficient of variation gave the following result:

$\begin{array}{ccc}\text { Mean of coefficient of variation } & \text { Under } 20 & 20 \text { and over } \\ \text { Standard deviation of coefficient } & 1.2 & 3.2 \\ \text { of variation } & 1.3 & 2.4\end{array}$

The difference between these two means was tested for reliability by determining the standard error of the difference:

$$
\frac{D}{\sigma D}=3.52,
$$

where $D$ equals the difference between the two means, and $\sigma D$, the standard error of that difference.

The result is indicative of a significant difference between these two groups, i.e., the chances of this being a true difference are over 1000 to 1 .

It would appear therefore that the dominant cortical frequency becomes less stable with increasing age, and this is demonstrable even in a series which contains no one over the age of 38 .

(d) Dominant frequency and the age factor. The total group of $\mathbf{5 0 0}$ young adults, between the ages of 17 and 47, was studied for correlation between their age and the actual frequency of their dominant rhythm at non-fasting blood sugar levels (in contrast to the consistency of this dominant frequency which has just been examined).

There have been several studies of this kind in relation to age in children (Berger (4), Loomis (5), Lindsley (6), Smith (7), and Weinbach (8)); but the age factor in adults has not received much attention. Bernhard and Skoglund $(9,10)$ demonstrated a difference of over half a cycle in the mean dominant frequency between two age groups of 15 to 18 and 19 to 30 , respectively.

In the present series of 500 adults between the ages of 17 and 47 , with a mean age of 24, the following results were obtained:

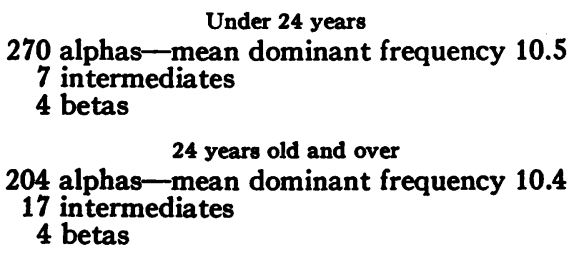

Thus, it would appear that age has no influence on the dominant frequency of the electro- encephalogram of adults up to the age of 47 . We have no data on normal individuals over this age.

(e) Dominant frequency and its relation to other physiological factors. The dominant frequencies in this group were examined for any possible correlation with sex, weight, height, or heightweight ratio, but no relation was found with any of these factors.

\section{(2) Percentage time alpha}

This is defined as the percentage time occupied by waves of 8.0 to 13.0 cycles per second, occurring either singly or in chains; i.e., it is the gross alpha count.

In 500 subjects who were examined at nonfasting blood sugar levels, the percentage time alpha varied in the group from 9 to 93 per cent, with a mean of 61 per cent. There was some alpha activity present in all records, even those which were predominantly beta in type.

The distribution among this group of the amount of alpha activity present at non-fasting blood sugar levels is given in the following distribution diagram (Figure 4).

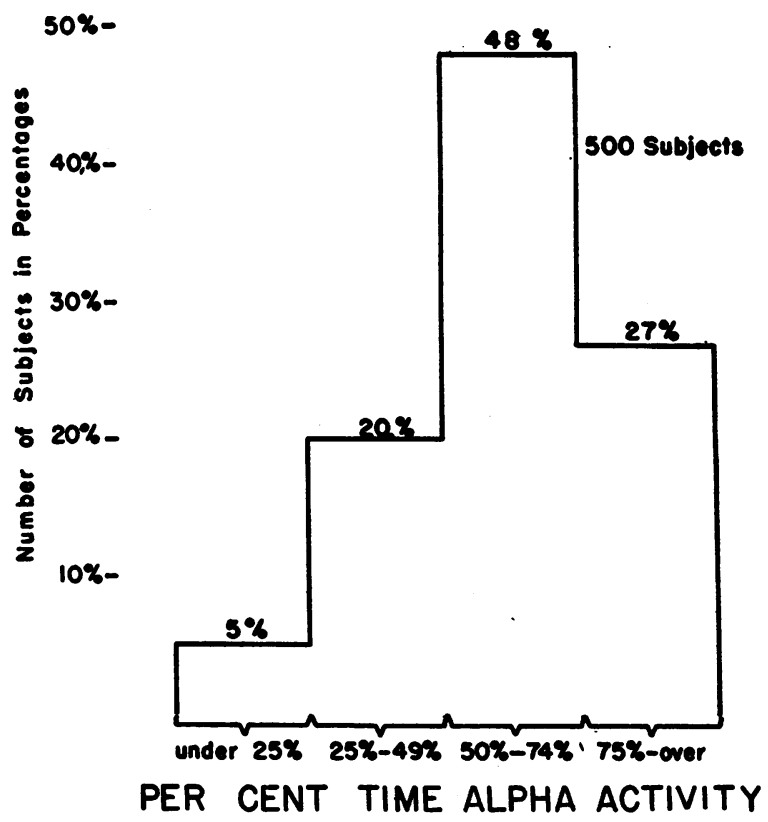

Fig. 4. Graph Illustrating 500 Normal Subjects (Expressed as Percentages) Grouped According to the Amount of Alpha Activity Present in Their RECORDS 
Of the 24 subjects with less than 25 per cent alpha activity, 16 had a predominantly intermediate rhythm, and 8 had dominant frequencies in the beta range.

(a) Consistency of percentage time alpha. The variability in percentage time alpha for an individual was not so marked in this series as has been described by Rubin (11) in bipolar recordings, the mean standard deviation for repeated tests on an individual being 7.1. It should be pointed out that in this work the percentage time alpha quoted includes all alpha activity present, whether occurring in single waves or in chains, whereas Rubin's observations are based on a criterion of 3 waves of alpha frequency occurring together; and in Rubin's experiments, the blood sugar was not controlled.

The relation between variability of percentage time alpha and high or low alpha percentage, as described by Rubin (12), did not hold in this series.

(b) Relation between percentage time alpha and dominant frequency. There was found to be an inverse relationship between percentage time alpha and the dominant frequency of that alpha; in other words, individuals whose percentage time alpha values were high showed dominant frequencies in the slower alpha range.

In the total series of $\mathbf{5 0 0}$ subjects, the records were examined for the relation between the dominant frequency of any alpha present and the percentage time occupied by this alpha activity. The following results were obtained:

\begin{tabular}{l|c|c|c}
\hline \hline Alpha frequency & $\begin{array}{c}\text { Number } \\
\text { of } \\
\text { subjects }\end{array}$ & $\begin{array}{c}\text { Mean } \\
\text { percentage } \\
\text { time } \\
\text { alpha }\end{array}$ & $\begin{array}{c}\text { Standard } \\
\text { deviation }\end{array}$ \\
\hline Slower than 10.5 cycles & 236 & 68.5 & 14.1 \\
10.5 cycles and faster & 264 & 54.7 & 20.1 \\
\hline
\end{tabular}

The difference between these 2 means was tested for reliability by determining the standard error of the difference which was found to be 8.90. Such a high standard error of the difference is beyond the possibility of chance.

It is therefore concluded that an inverse relationship exists between the dominant frequency of the alpha present and the amount of total alpha present.

(c) Relation between percentage time alpha and other physiological factors. No correlation was found in this series between the percentage time alpha and age, sex, height, weight, or the heightweight ratio.

\section{(3) Percentage time intermediate rhythm}

Frequencies of 13.5 to 17.5 cycles per second, i.e., those which lie between the alpha and beta ranges, are not commonly found in more than negligible quantities in the records of normal adults, and are only rarely found as the dominant frequency of the record. In this series of 500 subjects, there were 18 with a dominant frequency in this range, or 3.6 per cent.

Rhythms in this range, however, normally occur in bursts during the lighter stages of sleep (13), and it would not be surprising to find that they have a different physiological origin from the alpha waves. They do not appear to be merely accelerated alpha waves, since observations on sleep show that they appear abruptly and do not emerge by gradual transition from the higher alpha frequencies. These facts suggest that one is here dealing with a dichotomy.

As has already been noted, the electroencephalogram in normal adults contains very little activity in this intermediate frequency band. What little there is might be expected to appear in those records with the faster alpha frequencies as an extreme variation of their predominantly 12.5 to 13.0 cycle rhythms, but an examination of this series failed to establish any such correlation. There were no more waves of the intermediate frequencies in those records with dominant frequencies in the faster alpha range than in those with predominantly 9.0 and 9.5 cycle activity.

Unlike the so-called alpha and beta rhythms which are present to some extent in all records, the intermediate band of 13.5 to 17.5 cycle activity is sometimes totally absent, a fact which contributes to the impression, previously mentioned, that one is here dealing with a dichotomy.

\section{(4) Percentage time beta}

Beta activity (i.e., 18.0 cycles per second and over) was found, to a greater or less extent, in every record in this series at non-fasting blood sugar levels. As has already been remarked, all 


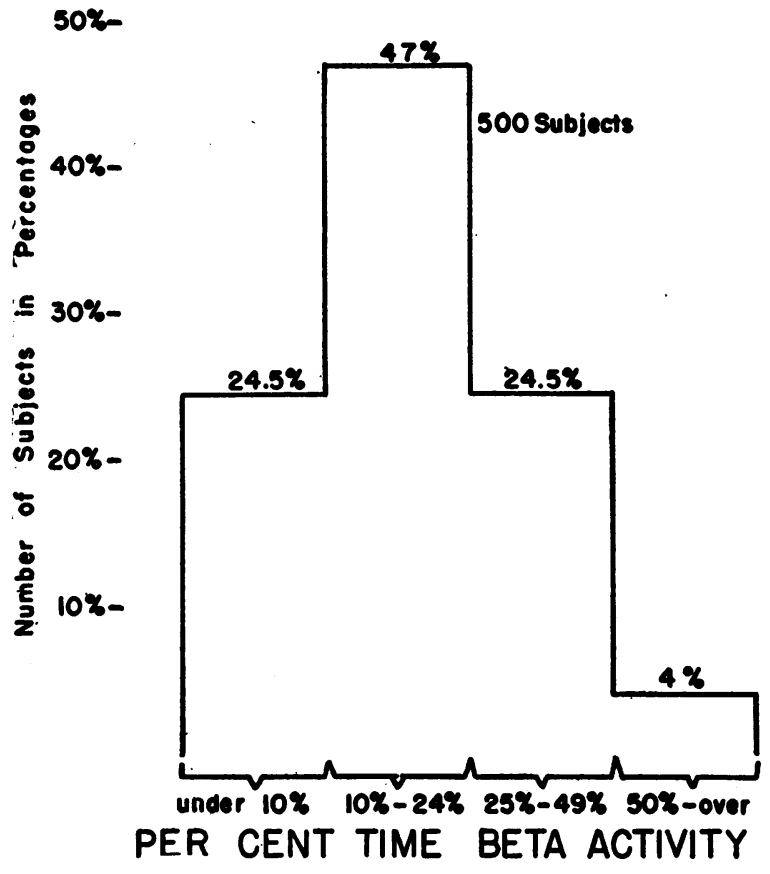

Fig. 5. Graph Showing 500 Normal Subjects (Expressed as Percentages) Grouped According to the Amount of Beta Activity Present in Their Records

electroencephalograms are a mixture of rhythms, and, in this series, the 8 cases which have been classified as beta type, all contained some alpha; their percentages of beta activity varied from 51 to 80 per cent. The distribution of beta activity in this series is given in Figure 5.

Figures 4 and 5 taken together do not, of course, give the total picture for the group, since the time occupied by intermediate frequencies and by slow activity is not represented. The amount of beta activity in an individual's record was found to be remarkably constant in repeated runs, and is characteristic for the individual. It did not vary with the degree of relaxation achieved by the subject.

Although in this series there was a tendency to more beta activity in the records of the older subjects, no statistical relation between it and age could be established. The amount of beta activity present did not correlate with sex, height, or weight.

\section{(5) Activity slower than 8.0 cycles per second}

Waves of a frequency slower than 8.0 cycles per second are here referred to as slow activity.
Traces of 7.0 to 7.5 per second frequencies were found in the records of 125 out of the series of 500 subjects, but only in 4 individuals was there more than 5 per cent of such slow activity in the 2-minute recording; of these 4 individuals, 1 had 19 per cent slow activity, 2 had 9 per cent, and 1 had 6 per cent.

Six-cycle waves were found in the occipital leads in 41 (8 per cent) of the 500 records at nonfasting blood sugar levels, but waves slower than 6 cycles per second were found in only 4 subjects, or less than 1 per cent.

Were an investigation of the potentials from the temporal regions to be made in a way similar to the present detailed study of the occipital potentials, it seems likely that there would be a higher incidence of 6- and 7-cycle waves in normal records, this being our experience and that of other electroencephalographers (14).

No wave in the range commonly called delta activity (i.e., 4 cycles or slower) was found.

\section{(6) Voltage}

This was measured by a pair of calipers, adjusted to the calibration made for voltage at the beginning of each record.

The maximum voltage was measured in every case and classified as to whether it was under $25 \mathrm{mV}$, over $25 \mathrm{mV}$ but under $50 \mathrm{mV}$, over 50 $\mathrm{mV}$ but under $100 \mathrm{mV}$, or over $100 \mathrm{mV}$. In the latter case, a further breakdown in classification was made between those who had less than 20 waves which reached $100 \mathrm{mV}$ in amplitude and those which had more than this number in a 2 minute run.

The distribution of voltage in this series of 500 normal subjects at non-fasting blood sugar levels was as follows:

\begin{tabular}{c|c|c|c|c|c}
\hline \hline Total & $\begin{array}{c}\text { Under } \\
25 \mathrm{mV}\end{array}$ & $\begin{array}{c}\text { Over } \\
25 \mathrm{mV} \\
\text { but under } \\
50 \mathrm{mV}\end{array}$ & $\begin{array}{c}\text { Over } \\
50 \mathrm{mV} \\
\text { but under } \\
75 \mathrm{mV}\end{array}$ & $\begin{array}{c}\text { Over } \\
75 \mathrm{mV} \\
\text { but under } \\
100 \mathrm{mV}\end{array}$ & $100 \mathrm{mV}$ \\
\hline 500 & 16 & 109 & 151 & 128 & 96 \\
\hline
\end{tabular}

(a) Voltage and dominant frequency. The faster frequencies tend to be of low voltage; no record with a dominant frequency faster than 11.5 had any potentials which reached as much as $100 \mathrm{mV}$.

In the alpha range, the records with the slower alpha frequencies were of higher voltage than 
the faster ones, thus following the usual character of oscillations in which the amplitude is inversely proportional to the frequency. In the following table, 474 normal subjects, whose dominant frequencies were in the 8.0 to 13.0 cycle band, are listed according to their voltage.

Maximum voltage under $50 \mathrm{mV}$ (105 subjects) Maximum voltage over $50 \mathrm{mV}$ (274 subjects) but under $100 \mathrm{mV}$

Maximum voltage over $100 \mathrm{mV}$ (95 subjects)

In this paper, a beginning has been made in an attempt to find what range of variation can be found for some of the characteristics of the electroencephalogram of normal adults. At the present stage, this study has been limited to an analysis of the cortical potentials from the occipital lobes, and it cannot be too strongly emphasized that a different set of data would undoubtedly be obtained from the frontal lobes, and different again from the temporal and parietal regions.

This study is also limited to analysis of electro(b) Voltage and percentage time alpha. The maximum voltage of the potentials in a record vary directly with the percentage time alpha activity present; i.e., those records which have a large amount of 8.0 to 13.0 cycle waves reach a higher maximum potential.

In the following table, voltage is related to the mean percentage time alpha found in the same series of 474 normal subjects whose dominant frequencies were in the 8.0 to 13.0 cycle band.

Maximum voltage

Under $25 \mathrm{mV}$

Over $25 \mathrm{mV}$, under $50 \mathrm{mV}$

Over $50 \mathrm{mV}$, under $100 \mathrm{mV}$

Over $100 \mathrm{mV}$
Mean percentage time alpha 40.3 (10 subjects) 48.4 (95 subjects) 65.4 (274 subjects) 73.6 (95 subjects)

\section{DISCUSSION}

Since the first development of electroencephalography, interest has been centered mainly on its application to clinical problems, and it is only recently that there has been a shift of interest to the study of the normal adult. For many reasons, it would have been preferable had the reverse taken place, for the development of this test to have proceeded from a basic study of the normal to a comparison of clinical records with a norm already well established.

The desirability is patent for the establishment of a yardstick for the normal population against which may be measured the variables found in pathological records. It is equally desirable in attempting to assess the electroencephalograms of normal subjects (as, for example, is being done in air-pilot selection) to have a quantitative basis from which one may calculate the chances of any observed phenomenon being a normal finding. encephalograms during normal breathing. A report of an investigation during hyperventilation will follow this, with special reference to the rôle of blood sugar and depth of hyperventilation.

In the past, the bulk of the work on both normal and clinical electroencephalograms has been done by the method of appraisal. The experienced electroencephalographer has looked at the record, compared it in his mind with his impression of those records which have previously come into his laboratory, and assessed it from this mental comparison. Where gross differences are present, such as are found in patients with epilepsy or with neoplasms, this method has in the main sufficed, but when finer shades of difference are being searched for, a more finely differentiated set of standards is necessary.

Such standards can only be set up on a basis of actual measurement, a method which is time absorbing, but essential in any research project designed to establish normal control standards. Were such a set of standards established on a large enough group of individuals, it would then be possible to estimate the chances of normality when any fine differences occur in the record, as for example, 7 cycle waves occurring singly in the occipital leads, or trains of 14 cycle waves. The percentage of normal records in which such waves occur would be known and the importance of the finding could thus be assessed.

A development in the measuring of electroencephalograms has been made by Gibbs and Grass (15) in the form of a spectrum analyzer. This apparatus gives a compilation of the amount of energy present at each frequency. At the present stage of our experience, we have found more meaning in the frequency of waves than 
in their voltage in normal records, and we therefore look for a method where the number of waves present at any frequency is not obscured by the voltage. An instrument for this purpose has recently been designed by Walter (16), but is not yet on the market.

It is obvious that the current differentiation of electrical potentials into alpha, beta, and delta rhythms is arbitrary and, in some respects, unfortunate. The classification of records into alpha and beta rhythms tends to obscure the fact that many so-called alpha records contain potentials of faster frequencies and that many beta records contain percentages of the slower alpha frequencies. A more accurate and more complete assessment could be made by describing the frequency distribution on the potentials. It would seem much wiser to describe rhythms in terms of the incidence of actually measured frequencies until some physiological or statistical reason can be found for grouping frequencies into certain rhythms.

The fact that significant contributions to clinical diagnosis. in epilepsy and the localization of neoplasms have been made by the crude methods of gross inspection of records would by no means invalidate the need for studies based upon careful measurements. However, once the data on a sufficiently large number of cases are collected, it might well be possible to develop simpler methods of analysis, based upon the known verifiable distributions of frequencies. This would seem a more logical approach and could give more precise information in the study of problems in which fine differentiations occur in the records.

\section{SUMMARY}

The occipital cortical potentials have been analyzed under conditions of controlled blood sugar in 500 subjects. Of these, the majority received but one test, but in 45 , repeated observations were obtained.

The following characteristics were found in these electroencephalograms.

1. The dominant frequency of an individual is comparatively stable, but becomes less so with increasing age. There is a statistically significant correlation between age and stability of dominant frequency (45 subjects).
2. Waves of 8.0 to 13.0 cycles per second ("alpha") were present in all records examined. The percentage time occupied by alpha waves varied inversely with the frequency of the dominant frequency. This inverse relationship has been established statistically in $\mathbf{5 0 0}$ subjects.

3. Waves of 13.5 to 17.5 cycles per second ("intermediate") are rarely found in any quantity in normal records, and constitute the dominant frequency in only 3.6 per cent of all normals examined (500).

4. Waves of 18.0 cycles per second and faster ("beta") were present in all records examined (500). The percentage time occupied by waves in this range is nearly constant for an individual in repeated runs (45 subjects).

5. Waves slower than 8.0 cycles per second are found in occipital potentials of 25 per cent of normal subjects. Waves as slow as 6.0 cycles were found in only 41 out of 500 subjects.

6. No waves of 4.0 cycles per second or slower ("delta") were found in the occipital recordings of any normal subject while breathing normally (500 subjects).

7. Maximum voltages are higher in those records which contain the most alpha activity, and in those records whose dominant frequencies fall in the slower alpha frequencies (500 subjects).

8. The physiological factors of sex, height, weight, or the height-weight ratio did not correlate with any characteristic in the brain wave record.

All the electroencephalographic tracings for this research were recorded in the Brain Wave Laboratory of the Massachusetts General Hospital with the cooperation of the director, Dr. Robert S. Schwab.

The authors are indebted to Mrs. Frances Cooperstein and Miss Margaret Gray for technical help, and to Mrs. Mary Newell for analysis of the records.

\section{BIBLIOGRAPHY}

1. Loomis, A. L., Harvey, E. N., and Hobart, G., Electrical potentials of the human brain. J. Exper. Psychol., 1936, 19, 249.

2. Jasper, H. H., and Cruikshank, R. M., Visual stimulation and the after-image as affecting the occipital alpha rhythm. J. Gen. Psychol., 1937, 17, 29.

3. Jasper, H. H., and Andrews, H. L., Electroencephalography. Arch. Neurol. and Psychiat., 1938, 39, 96.

4. Berger, H., Über das Elektrenkephalogramm des Menschen. Arch. f. Psychiat., 1932, 98, 231. 
5. Loomis, A. L., Harvey, E. N., and Hobart, G. A., Cerebral states during sleep as studied by human brain potentials. J. Exper. Psychol., 1937, 21, 127.

6. Lindsley, D. B., Brain potentials in children and adults. Science, 1936, 84, 354.

7. Smith, J. R., Electroencephalogram during infancy and childhood. Proc. Soc. Exper. Biol. and Med., 1937, 36, 384.

8. Weinbach, A. P., Contour maps, center of gravity, moment of inertia and surface area of human body. Human Biol., 1938, 10, 356.

9. Bernhard, C. G., and Skoglund, C. R., Recherches sur la fréquence alpha de l'électro-encéphalogramme chez l'enfant. Acta psychiat. et neurol., 1939, 14, 223.

10. Bernhard, C. G., and Skoglund, C. R., On alpha fre- quency of human brain potentials as function of age. Skandinav. Archiv. f. Physiol., 1939, 82, 178.

11. Rubin, M. A., The distribution of the alpha rhythm over the cerebral cortex of normal man. J. Neurophysiol., 1938, 1, 313.

12. Rubin, M. A., A variability study of the normal and schizophrenic occipital alpha rhythm. J. Psychology, 1938, 6, 325.

13. Loomis, A. L., Harvey, E. N., and Hobart, G., Further observations on potential rhythms of cerebral cortex during sleep. Science, 1935, 82, 198.

14. Kershman, J., Personal communication.

15. Grass, A. M., and Gibbs, F. A., Fourier transform of electroencephalogram. J. Neurophysiol., 1938, 1, 521.

16. Walter, W. G., An automatic low frequency analyzer. Electronic Engineering. June 1943. 